\title{
Plant Age Affects Root Infection and Development of Foliar Symptoms of Soybean Sudden Death Syndrome
}

\author{
C. C. Gongora-Canul and L. F. S. Leandro, Department of Plant Pathology, Iowa State University, Ames 50010
}

\begin{abstract}
Gongora-Canul, C. C., and Leandro, L. F. S. 2011. Plant age affects root infection and development of foliar symptoms of soybean sudden death syndrome. Plant Dis. 95:242-247.

Soybean sudden death syndrome is characterized by root rot followed by the development of foliar symptoms. However, it is not known how time of infection affects disease severity. Soybean plants were inoculated at $0,4,7,14,21,28$, and 35 days after planting (DAP) by drenching potting media with conidia of Fusarium virguliforme, then incubating in growth chambers at $17^{\circ} \mathrm{C}$ for 7 days followed by $24^{\circ} \mathrm{C}$ for 31 days. Root rot and severity of foliar symptoms were assessed 18 and 38 days after inoculation (DAI). Root rot developed on plants inoculated at all ages but plants inoculated at seed stage (0 DAP) had the

highest $(P<0.01)$ root rot severity $(>90 \%)$. At 38 DAI, foliar symptoms were severe $(>80 \%)$ on plants inoculated at 0 DAP but did not develop on plants inoculated at all other ages. Xylem colonization by $F$. virguliforme was more frequent in plants inoculated at 0 DAP than on plants inoculated at later stages. The results of this study suggest that soybean roots become less susceptible to xylem colonization and the subsequent development of foliar symptoms as plants mature. Therefore, practices aimed at protecting seed and seedling roots from infection may improve soybean sudden death management.
\end{abstract}

Sudden death syndrome (SDS) of soybean (Glycine max (L.) Merr.), caused by Fusarium virguliforme (O'Donnel \& T. Aoki) $(1,2)$, can cause significant yield losses $(33)$. The symptoms of the disease include root rot and interveinal chlorosis and necrosis of the leaves (24), the latter caused by pathogen toxins translocated from the roots $(13,14,16,24,25)$. Foliar symptoms typically appear at or after flowering (24) but root infections can occur in early stages of root development (23). For example, Gao et al. (7) demonstrated that roots of young soybean seedlings were colonized by F. virguliforme within 2 weeks after emergence in field soil. Huang and Hartman (11) isolated the fungus within 3 to 10 days after inoculation in a controlled environment. Observations that earlyseason planting of soybean tends to increase SDS severity $(10,32)$ suggest that seedling infection is most conducive to development of foliar symptoms later in the season. However, it is not known whether soybean susceptibility to SDS varies with growth stages, or how the timing of infection affects disease severity.

The age at which plant roots are inoculated can affect severity of other diseases $(8,15,29)$. Kuruppu et al. (15) found that 1-week-old plants were more susceptible to red crown rot of soybean caused by Calonectria illicicola than older plants. Similarly, soybean plants became less susceptible to Diaporthe stem canker as plant age increased (29) but the opposite was observed with soybean infected by Phialophora gregata (20). For SDS, defining the period of susceptibility for root infections and subsequent foliar symptoms may prove useful in understanding the etiology of this disease and contribute to more effective management. Our hypothesis is that soybean plants become less susceptible to root infection conducive to SDS foliar symptoms with increasing plant age at time of infection.

Clarification of how susceptibility to SDS varies with plant age may have implications for disease management decisions, such as application of seedling protectant fungicides, as well as for resistance breeding, through the identification of the key growth stages to search for resistance mechanisms. The objective of this work

Corresponding author: L. F. S. Leandro, E-mail: 1leandro@iastate.edu

Accepted for publication 1 November 2010.

doi:10.1094/PDIS-06-10-0448

(C) 2011 The American Phytopathological Society was to determine whether plant age affects the ability of $F$. virguliforme to infect roots and cause SDS foliar symptoms.

\section{Materials and Methods}

Plant material and inoculum. Susceptible soybean cv. AG2403 (Asgrow Seed, St. Louis), a glyphosate-tolerant, maturity group II cultivar, was used in all experiments. The inoculum source was a single-spore isolate of F. virguliforme (LL0009) obtained in 2006 from roots of a soybean plant with typical SDS foliar symptoms from Nevada, IA. The isolate was initially obtained on modified Nash and Snyder medium (MNSM; 4) and then stored on carnation leaf agar at $4^{\circ} \mathrm{C}$. Before inoculation, cultures of the isolate were grown on potato dextrose agar at room temperature $\left(24 \pm 2^{\circ} \mathrm{C}\right)$ in darkness for 12 days. Conidial suspensions were prepared by flooding the cultures with sterile deionized water (SDW), dislodging the conidia with a rubber policeman, then filtering through three layers of sterile cheese cloth; SDW was used to adjust the density to $6 \times 10^{3}$ conidia $/ \mathrm{ml}$.

Growth-chamber experiment. An experiment was established to test the effect on plant age on severity of SDS root rot and foliar symptoms. A randomized complete block design encompassed seven plant ages at the time of inoculation $(0,4,7,14,21,28$, and 35 days after planting [DAP]), two sampling times (18 and 38 days after inoculation [DAI]), and five replications (12-cm-diameter pots with three seeds each). To obtain plants of different ages at inoculation, soybean seed were planted over a 5-week period. Before planting, soybean seed were surface disinfested by immersion in $0.5 \%$ sodium hypochlorite for $2 \mathrm{~min}$ and rinsed twice in SDW. Seed were planted in pasteurized soil and sand mix $(1: 1, \mathrm{vol} / \mathrm{vol})$ amended with $1.5 \%$ (wt/wt) sterilized cornmeal to facilitate homogenous colonization of the soil by $F$. virguliforme. After planting, pots were maintained in a growth chamber at $24^{\circ} \mathrm{C}$ under a 14-h photoperiod.

On the day of inoculation, soybean growth stages (6) ranged from seed to V5 (Table 1). Each pot was drenched with $130 \mathrm{ml}$ of the $F$. virguliforme conidial suspension, resulting in an initial concentration of approximately 800 conidia/g of soil. After inoculation, plants were exposed to $17 \pm 2{ }^{\circ} \mathrm{C}$ for 7 days to induce root infection, followed by $24 \pm 2{ }^{\circ} \mathrm{C}$ for 31 days to induce foliar symptoms (28). The experiment was repeated once; the 4-DAP plant age treatment was included only in the second run of the experiment.

At 18 and 38 DAI, after the root systems were gently washed in running tap water, root rot severity was visually rated as the per- 
centage of root length showing brown or black discoloration. Severity of foliar symptoms was rated as the percentage of the total leaf area of each plant exhibiting chlorosis and necrosis typical of SDS. Frequency of root colonization by $F$. virguliforme was calculated by culturing 1-cm-long root pieces on MSNM (4). Two root pieces were arbitrarily excised from tap and lateral roots of each of the three plants per pot. The plates were incubated at $24 \pm 2^{\circ} \mathrm{C}$ in the darkness, and the percentage of root pieces showing blue or purple colonies characteristic of $F$. virguliforme was counted 5 to 10 days later (25).

Root colonization experiment. The infection process of $F$. virguliforme was compared on soybean plants inoculated at $0,4,7$, and 14 DAP. Plants were grown in growth chambers and inoculated as described above. For each plant age and sampling time, six plants were inoculated and three plants were used as noninoculated controls. Root rot severity and foliar disease severity were evaluated 17 and 27 DAI, after roots were washed in running tap water. Three 1-cm-long root pieces were then arbitrarily excised from the top, middle, and bottom of the tap root of each plant. The 18 root pieces were pooled for each plant age and sampling time combination, and fixed in formalin-acetic acid-alcohol from 2 to 4 days at $4^{\circ} \mathrm{C}$. Ten arbitrarily selected root pieces from each sample set were then dehydrated in a graded ethanol series ( $1 \mathrm{~h}$ each step at 50, 70, 8, 9 , and $100 \%)$, cleared with xylene $(100 \%)$, and infiltrated and embedded using paraplast paraffin (Fisher Scientific, Pittsburgh). Sections were cut at $8 \mu \mathrm{m}$ of thickness using an A/O 820 rotary microtome (Fisher Scientific), then mounted onto glass slides, deparaffinized, stained with $1 \%$ toluidine blue in $1 \%$ borax, dehydrated in graded ethanol solution, and cleared with xylene. Five sections of each of the 10 root pieces were observed using a Zeiss AxioPlan II Imaging compound microscope (Carl Zeiss Inc., Thornwood, NY) at $\times 400$ magnification. Presence or absence of $F$. virguliforme hyphae in xylem and cortical root tissues was recorded on a binary scale, and the frequency of xylem and cortex colonization was estimated as the proportion of root pieces showing hyphae.

Field experiment. A microplot trial was established in 2008 and 2009 at the Iowa State University Hinds Research Farm near Ames to determine whether the effect of plant age observed in growthchamber trials also occurred under field conditions. The field plot had been cropped with continuous soybean for 6 years, and had a history of SDS due to natural and artificial infestations. A completely randomized design was used with four plant age treatments and 10 replicate plants per treatment.

In order to obtain plants that were $0,4,7$, and 14 days old at the time of inoculation, soybean seed were planted over a 2-week period in 8-cm-diameter styrofoam cups containing $280 \mathrm{~g}$ of potting medium, as described above. On 24 May 2008 and 4 May 2009, plants were removed from the styrofoam cups and transplanted into 10-cm-deep holes, spaced $25 \mathrm{~cm}$ apart between and within rows. Immediately after transplanting, $100 \mathrm{ml}$ of an inoculum suspension $\left(5 \times 10^{4}\right.$ conidia $\left./ \mathrm{ml}\right)$ was applied around the root zone of each plant to achieve an approximate inoculum concentration of $1.8 \times 10^{4}$ conidia/g of soil in the volume of soil contained in the holes. Plants were watered as needed to maintain adequate soil moisture throughout the experiment. Severity and incidence of SDS foliar symptoms were evaluated weekly until senescence using the methods described above.

Statistical analysis. Analysis of variance was performed with the PROC GLM procedure of SAS (SAS Institute, Cary, NC). Two repetitions of the growth-chamber experiment were analyzed separately due to a significant interaction of experiment-plant age and the lack of the 4-DAP plant age treatment in the first run. The interaction of analyzed time-plant age at inoculation was evaluated using the "slice" option in SAS. The Tukey-Kramer procedure was used to test differences in root and foliar severity and frequency of root colonization by $F$. virguliforme. For the field experiment, the area under disease progress curve (AUDPC) for foliar severity was calculated for each plant age treatment by using the trapezoidal integration method (3). To detect differences in foliar severity AUDPC, the Tukey-Kramer procedure was used ( $\alpha$ $=0.05)$.

\section{Results}

Growth-chamber experiment. Plants inoculated at all ages developed root rot but differences $(P<0.01)$ in root rot severity were detected among plant age treatments in each trial (Fig. 1). At 18 DAI, root rot severity was greatest in plants inoculated at 0 DAP (>75\%) and ranged from 7.6 to $40.3 \%$ at older plant ages. At 38 DAI, plants inoculated at 0 DAP showed severe root rot $(>94 \%)$, whereas root rot severity on plants inoculated at 4 DAP or older ranged from 25 to $57 \%$ and did not differ significantly among treatments (Fig. 1A and D).

Differences in severity of SDS foliar symptoms were significant $(P<0.01)$ among plant ages. At both sampling times, only plants inoculated at 0 DAP developed foliar symptoms, whereas plants inoculated at 4 DAP or later never developed foliar symptoms (Fig. $1 \mathrm{~B}$ and $\mathrm{E})$. The growth stage of plants evaluated 38 DAI ranged from V2 to R2 (Table 1). Frequency of $F$. virguliforme isolation from soybean roots differed among plant ages at both sampling times $(P<0.01)$, except at $18 \mathrm{DAI}$ in the first experiment. At both 18 and $38 \mathrm{DAI}$, the pathogen was isolated more frequently from plants inoculated at 0 DAP than those inoculated at later ages (Fig. $1 \mathrm{C}$ and $\mathrm{F}$ ).

Root colonization experiment. On plants inoculated to compare the infection process of $F$. virguliforme, plant age affected severity of root rot and foliar symptoms (data not shown) in a manner parallel to the results of the growth-chamber experiment described above. Hyphae of $F$. virguliforme were observed in roots of all inoculated plants but hyphae were never observed in noninoculated plants (Fig. 2). At 17 DAI, hyphae were observed in the root cortex of all inoculated plants, with frequency ranging from 70 to $94 \%$ of root pieces. Colonization of the xylem was $<5 \%$ in roots of plants inoculated at 4 and 7 DAP (Fig. 3A), and these values did not differ significantly from zero $(P=0.53)$.

At 27 DAI, hyphae formed dense networks in the cortical tissue of plants inoculated at all ages (Fig. 2B). Frequency of cortex colonization ranged from 82 to $98 \%$ and did not differ among plant ages. Hyphae were observed forming intercellular and extracellular networks in the xylem of $56.5 \%$ of roots pieces from plants inoculated at 0 DAP (Figs. 3B and 2C), significantly greater than for the other age treatments. Xylem colonization was also observed in plants inoculated at 14 DAP but at a frequency (14\%) that was not significantly different from plant ages where no xylem colonization was observed.

Field experiment. Only results from the 2008 field trial are presented because SDS symptoms did not develop in 2009. In the 2008 trial, progress of SDS foliar severity differed significantly among plants inoculated at different ages. Two phases of foliar

Table 1. Soybean growth stage (GS) at time of inoculation and at 18 and 38 days after inoculation (DAI) for soybean plants (cv. AG2403) inoculated at seven different ages with a conidial suspension of Fusarium virguliforme and grown at $24^{\circ} \mathrm{C}$ in growth-chamber conditions for the two repetitions of the experiment

\begin{tabular}{llcccccc}
\hline & \multicolumn{7}{c}{ GS for plant age (days) at inoculation } \\
\cline { 2 - 7 } GS & $\mathbf{0}$ & $\mathbf{4}$ & $\mathbf{7}$ & $\mathbf{1 4}$ & $\mathbf{2 1}$ & $\mathbf{2 8}$ & V3 \\
\hline At inoculation & Seed & VE & VC & V1-V2 & V2 & V4-V5 \\
At first sampling date (18 DAI) & V1 & V2 & V2-V3 & V3-V4 & V4-V5 & V5 \\
At second sampling date (38 DAI) & V2-V3 & V4 & V5 & V6 & V7-V8 & V8-R1 & V6-V7 \\
\hline
\end{tabular}


symptoms were observed (Fig. 4). The first phase, at vegetative stages V2 to V4 (26 to $58 \mathrm{DAI}$ ), was followed by a remission of foliar symptoms due to defoliation of symptomatic leaves and production of new leaves. A second phase of disease occurred at reproductive stages R5 to R7, starting 79 DAI. For the first phase of foliar symptoms, plants inoculated at 0 DAP showed significantly greater AUDPC than all other plant age treatments $(P<0.0004)$, whereas plants inoculated at 4 DAP showed low foliar severity AUDPC and those inoculated at 7 and 14 DAP had no foliar symptoms (Table 2). In the second phase, however, plants inoculated at all ages developed foliar symptoms and no significant differences in foliar severity AUDPC were observed among plant age treatments $(P=0.14)$. Total AUDPC calculated for the experiment was greatest $(P=0.0004)$ for the 0 -DAP plant age treatment and did not differ significantly among the older plant ages (Table 2).

\section{Discussion}

This is the first study to document that plant age at time of inoculation influences susceptibility to SDS of soybean. In growthchamber trials, severity of root rot and foliar symptoms was reduced by increasing plant age at the time of inoculation. Although root rot developed on plants inoculated at all plant ages, foliar symptoms developed only on plants inoculated at the seed stage $(0$ DAP). Plants in field conditions also showed the most severe foliar symptoms when inoculated at seed stage. These findings indicate
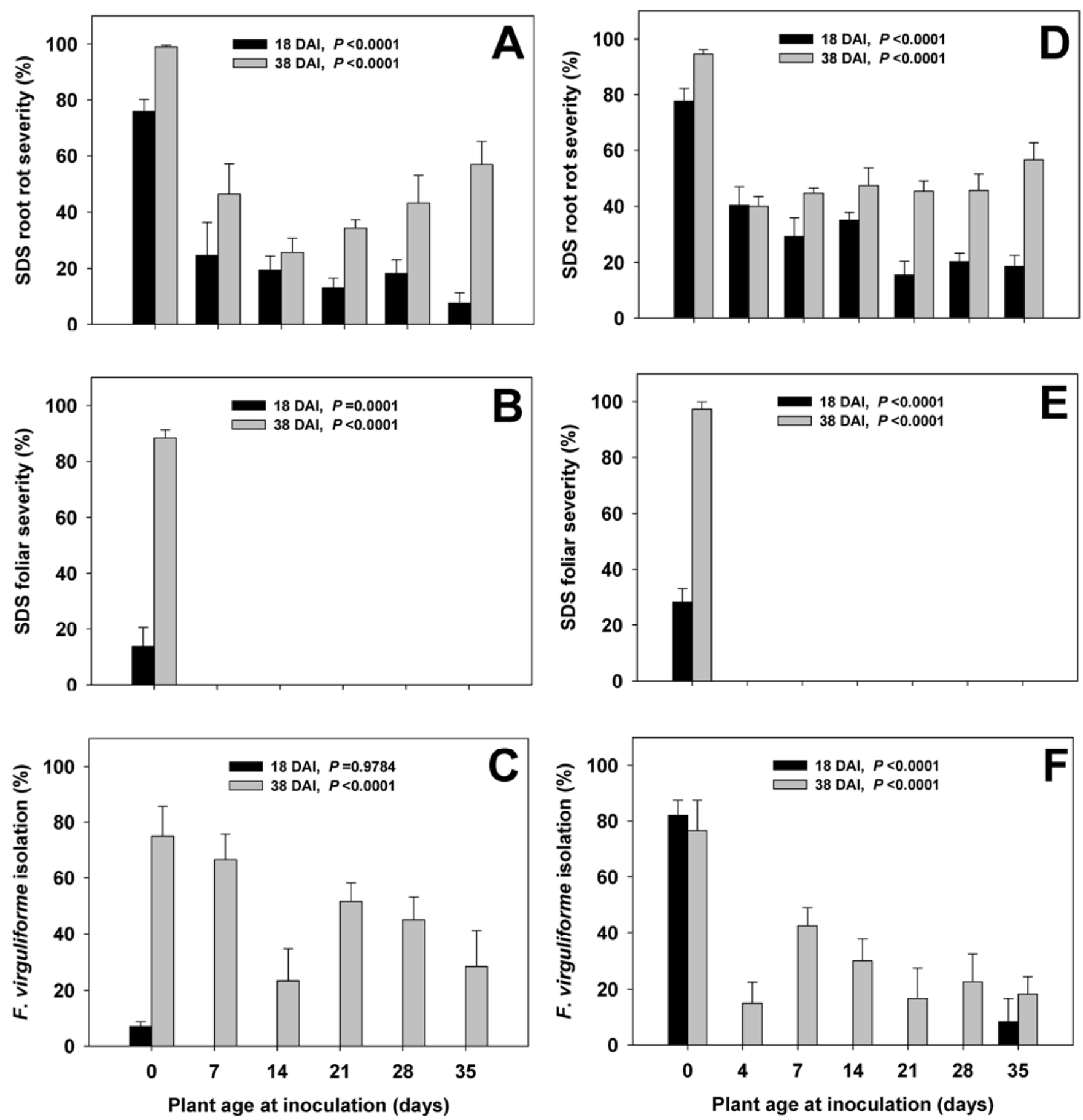

Fig. 1. Severity of root rot and foliar symptoms of sudden death syndrome and frequency of isolation of Fusarium virguliforme from roots of soybean plants inoculated at different ages with $F$. virguliforme and evaluated 18 and 38 days after inoculation (DAl) in a growth-chamber experiment. Data represent repetition $\mathbf{A}, \mathbf{B}$, and $\mathbf{C}$, one and $\mathrm{D}, \mathrm{E}$, and $\mathbf{F}$, two of the experiment ( $n=15$ plants/age/sampling time). 
that post-infection development of foliar symptoms is greatly dependent on plant age at the time of infection. Plant age also impacts disease development incited by other soilborne pathogens. For example, soybean roots were most susceptible to red crown rot caused by $C$. ilicicola during the first week after seedling emergence (15), and younger celery plants developed more severe disease than older plants when infected by $F$. oxysporum f. sp. apii (8). In contrast, older plants were more susceptible to brown stem rot of soybean caused by $P$. gregata (20) and root rot of cereals caused by Cochliobolus sativus (31).

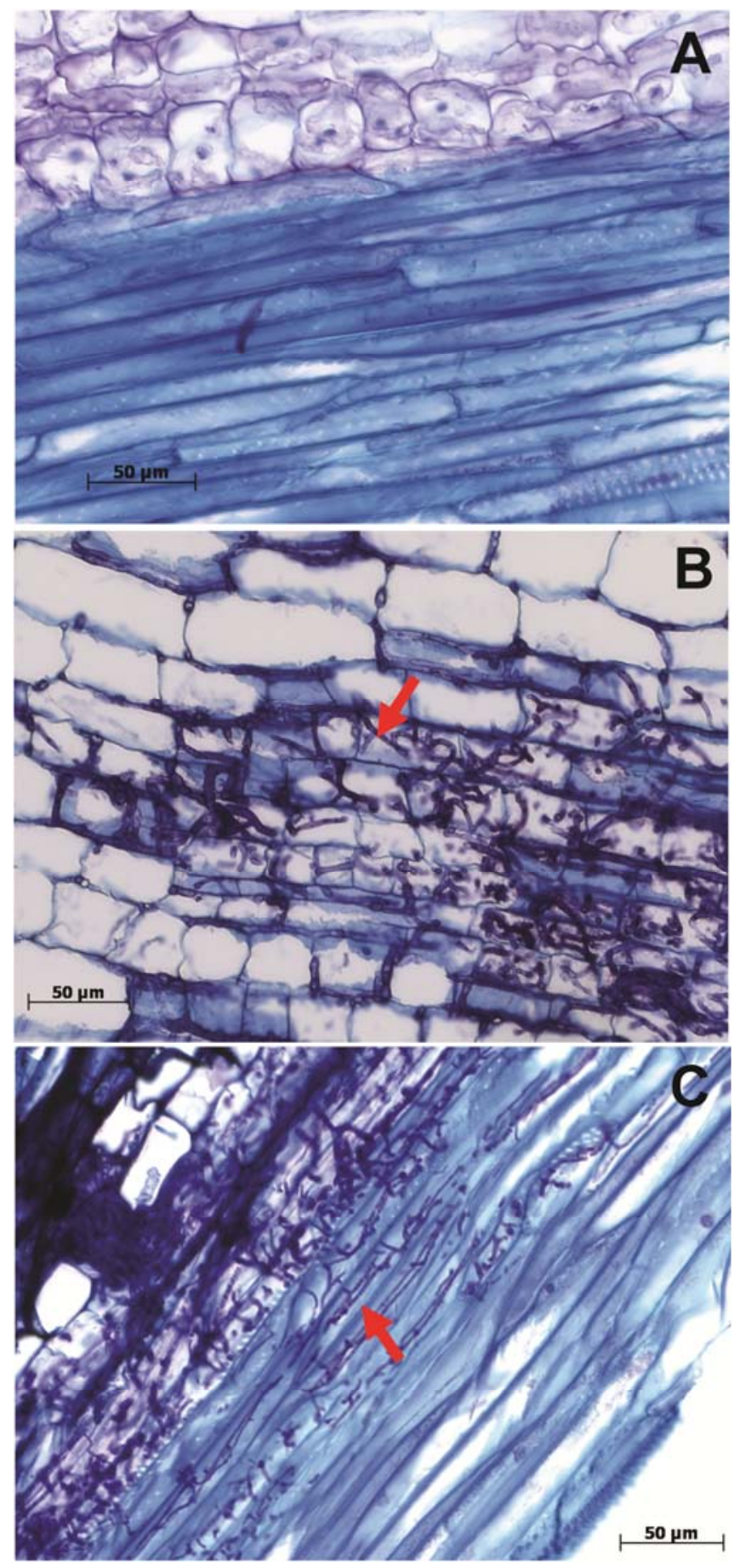

Fig. 2. Hyphal colonization of soybean roots inoculated with Fusarium virguliforme at different plant ages and observed 27 days after inoculation. A, Healthy cortical and vascular tissue of a noninoculated plant; $\mathbf{B}$, intra- and intercellular colonization of the cortical tissue cells in a plant inoculated at 4 days after planting (DAP); and $\mathrm{C}$, hyphae in the cortex and xylem tissues of a plant inoculated at seed stage $(0$ DAP). Roots were observed at a magnification of $\times 400$ using an optical microscope.
In this current study, root rot severity and frequency of $F$. virguliforme isolation from roots was greatest in plants inoculated at seed stage and tended to decrease on progressively older plants at inoculation, suggesting that soybean roots are most susceptible to infection at early seedling stages. Several mechanisms could be involved in the decreased susceptibility of older roots. For example, higher suberin content in older sections of soybean roots has
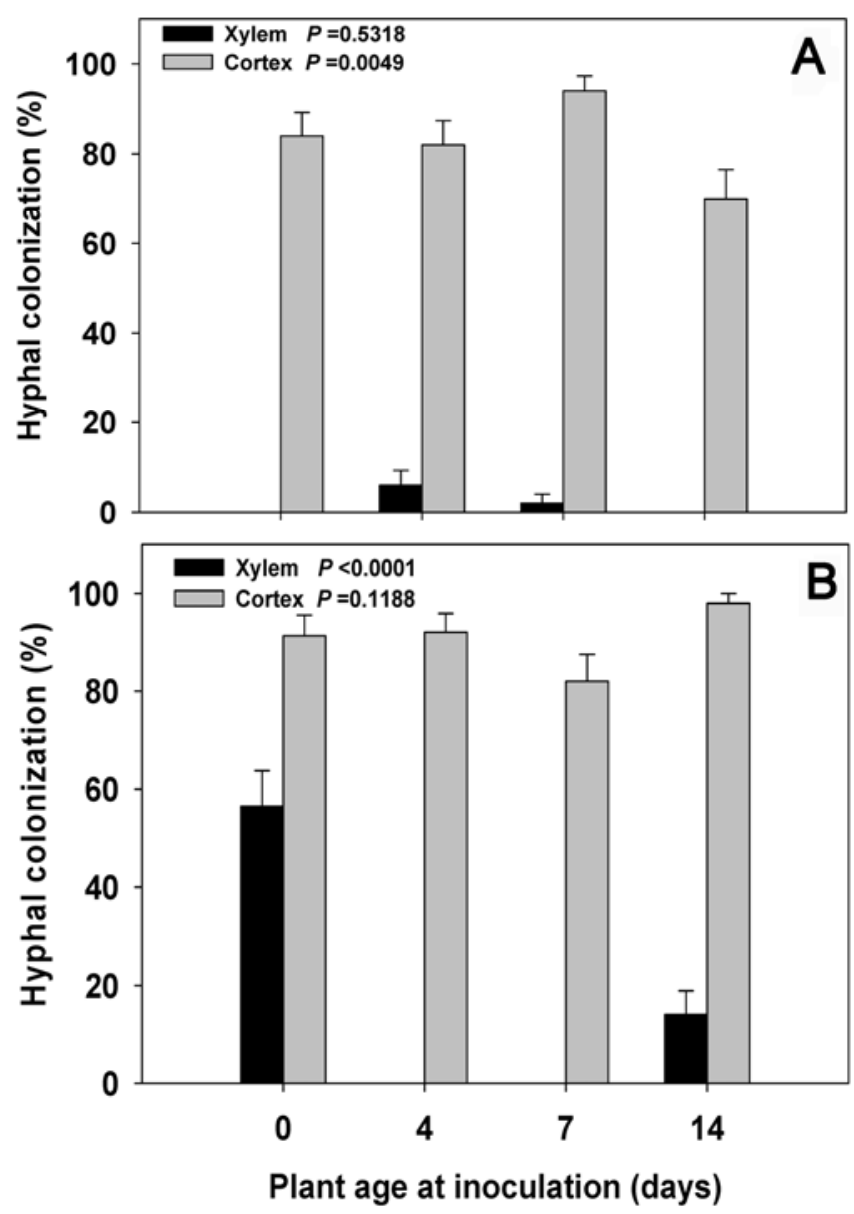

Fig. 3. Frequency of colonization (\%) by Fusarium virguliforme hyphae of cortical and xylem root tissues of soybean plants inoculated at four different ages and sampled A, 17 and B, 27 days after inoculation. Five longitudinal sections from each of 10 root pieces per plant age treatment were observed at each sample time $(n=50)$.

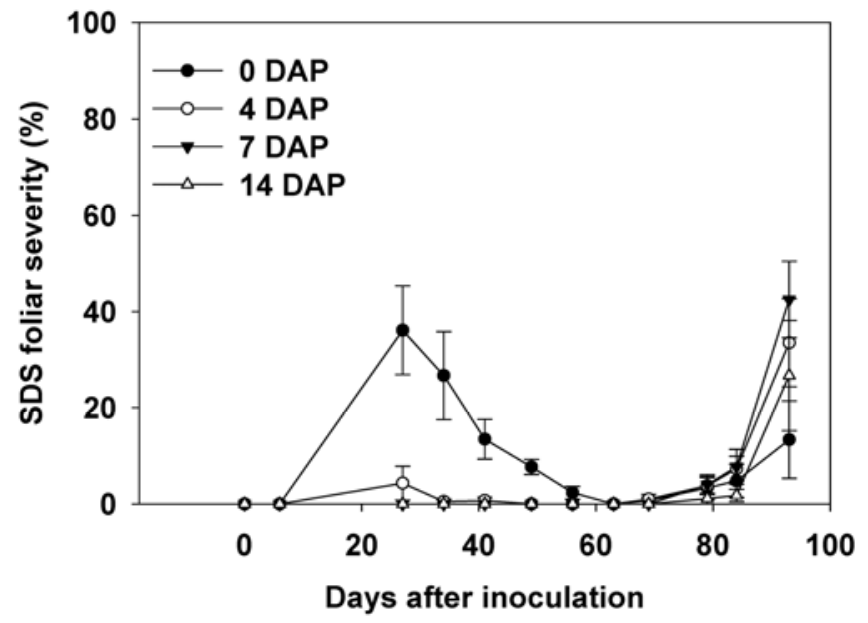

Fig. 4. Progress of sudden death syndrome foliar severity on soybean plants inoculated at four different ages (DAP =days after planting) on the day of transplanting into a field plot in lowa in $2008(n=10)$. 
been associated with increased resistance to infection by Phytophthora sojae and is thought to play a role in partial resistance to this pathogen $(21,30)$. Suberin can be synthesized within 2 to 6 days after soybean inoculation with $P$. sojae (21); therefore, it is possible that suberin accumulation during this period contributed to the sharp differences in foliar symptoms observed between plants inoculated at 0 and 4 DAP. Flavones (12) and phytoalexins, such as glyceollin (17), may also be involved in root resistance to $F$. virguliforme in soybean, and the concentration of isoflavonoids are known to increase within days after inoculation with other pathogens (5).

The higher frequency of $F$. virguliforme observed in the xylem of plants inoculated at 0 DAP compared with plants inoculated at older ages suggests that the mechanisms involved may restrict colonization of the plant vascular system, thereby limiting toxin movement to the leaves (19) and preventing the development of foliar symptoms. Higher susceptibility of plants inoculated at 0 DAP may be related to reduced lignification of the root cell wall of seedlings compared with root cell walls of older plants (17). Because $F$. virguliforme is considered an efficient lignin degrader $(18,22)$, further studies to clarify the role of lignin on the infection process of this fungus would be valuable.

In field conditions, SDS foliar symptoms usually develop during plant reproductive stages (24). In our growth-chamber studies, however, plants inoculated at 28 and 35 DAP did not develop SDS foliar symptoms even though they were at the R1 to R2 growth stage by the end of the experiments. Because disease progress was not monitored in later reproductive stages, it is not possible to conclude that plants would have remained asymptomatic if they were maintained for a longer period of time. However, results of the field experiment suggest that there are two phases of development of SDS foliar symptoms: a first phase during vegetative stages that is followed by remission of symptoms and a second phase during late reproductive stages. This dual phase of disease has been observed in other SDS field trials $(26,27)$. Remission of SDS foliar symptoms has been attributed to dry soil conditions (9) but drought stress was not a limiting factor in the trials reported in this study because plots were irrigated. Furthermore, a similar remission in foliar symptoms was observed by the authors in greenhouse studies where plants were watered daily.

It is not clear whether the second period of foliar symptom expression observed in this study resulted from new root infections during plant reproductive stages or from infections that occurred during vegetative growth stages that remained latent until the second phase of the disease. It is possible that foliar symptoms may have developed in plants inoculated at older ages if exposed to different environmental conditions or inoculum density or with plants of different genotypes. However, because soybean plants were most susceptible to SDS when inoculated at the seed stage, practices aimed at protecting seedlings should be investigated as a disease management option. In addition, studies on gene expression during early stages of root development and quantification of

Table 2. Area under the disease progress curve for foliar severity of sudden death syndrome during vegetative and reproductive growth periods on soybean plants (cv. AG2403) inoculated with Fusarium virguliforme at four different ages after transplanting into field conditions in 2008

\begin{tabular}{lccc}
\hline Plant age & \multicolumn{3}{c}{ Growth period $^{\mathbf{y}}$} \\
\cline { 2 - 4 }$(\mathbf{D A P})^{\mathbf{z}}$ & $\begin{array}{c}\text { Vegetative } \\
\mathbf{( 0 - 6 3 ~ D A I )}\end{array}$ & $\begin{array}{c}\text { Reproductive } \\
(\mathbf{6 3 - 9 3} \text { DAI) }\end{array}$ & $\begin{array}{c}\text { Total } \\
\mathbf{( 0 - 9 3 ~ D A I ) ~}\end{array}$ \\
\hline 0 & $780.2 \mathrm{a}$ & $112.2 \mathrm{a}$ & $892.4 \mathrm{a}$ \\
4 & $69.0 \mathrm{~b}$ & $238.3 \mathrm{a}$ & $307.3 \mathrm{~b}$ \\
7 & $0.0 \mathrm{~b}$ & $275.1 \mathrm{a}$ & $275.2 \mathrm{~b}$ \\
14 & $0.0 \mathrm{~b}$ & $96.1 \mathrm{a}$ & $96.1 \mathrm{~b}$ \\
$P>F$ & $P<0.0001$ & $P=0.1409$ & $P<0.0004$ \\
\hline
\end{tabular}

${ }^{\mathrm{y}} \mathrm{DAI}=$ number of days after inoculation.

${ }^{\mathrm{z}}$ Plant age at inoculation $=$ number of days after planting (DAP) when plants were inoculated. Plants were grown in pots in the greenhouse and inoculated on the day of transplanting into a field plot. suberinization and phytoalexin production may clarify the mechanisms of root resistance to SDS.

\section{Acknowledgments}

This study was funded by the Iowa Soybean Association and the North Central Soybean Research Program. We thank T. Kaspar, United States Department of Agriculture-Agricultural Research Service National Soil Tilth Laboratory, Ames, IA, for advice on root growth and physiology; and H. Horner and T. Pepper of the Iowa State University Microscopy and Nanoimaging Facility for their assistance with microscopy.

\section{Literature Cited}

1. Aoki, T., O’Donnell, K., Homma, Y., and Lattanzi, A. R. 2003. Sudden death syndrome of soybean is caused by two morphologically and phylogenetically distinct species within the Fusarium solani species complex-F. virguliforme in North America and F. tucumaniae in South America. Mycologia 95:660-684.

2. Aoki, T., O'Donnell, K., and Scandiani, M. M. 2005. Sudden death syndrome of soybean in South America is caused by four species of Fusarium: Fusarium brasiliense sp. nov., F. cuneirostrum sp. nov., F. tucumaniae, and F. virguliforme. Mycoscience 46:162-183.

3. Campbell, C. L., and Madden, L. V. 1990. Introduction to Plant Disease Epidemiology. John Wiley \& Sons, New York.

4. Cho, J. H., Rupe, J. C., Cummings, M. S., and Gbur, E. E., Jr. 2001. Isolation and identification of Fusarium solani f. sp. glycines from soil on modified Nash and Snyder's medium. Plant Dis. 85:256-260.

5. Cho, M.-J., and, Harper, J. E. 1991. Effect of inoculation and nitrogen on isoflavonoid concentration in wild-type and nodulation-mutant soybean roots. Plant Physiol. 95:435-442.

6. Fehr, W. R., Caviness, C. E., Burmood, D. T., and Pennington, J. S. 1971 Stage of development descriptions for soybeans, Glycine max (L.) Merrill. Crop Sci. 11:929-931.

7. Gao, X., Hartman, G., and Niblack, T. 2006. Early infection of soybean roots by Fusarium solani f. sp. glycines. (Abstr.). Phytopathology 96:S38.

8. Hart, L. P., and Endo, R. M. 1981. The effect of length of exposure to inoculum, plant age, root development, and root wounding on Fusarium yellows of celery. Phytopathology 71:77-79.

9. Hershman, D. E. 1990. Soybean sudden death syndrome in Kentucky. Univ. Ky. Coop. Ext. Serv. Inf. Bull. PPA-37.

10. Hershman, D. E., Hendrix, J. W., Stuckey, R. E., Bachi, P. R., and Henson, G. 1990. Influence of planting date and cultivar on soybean sudden death syndrome in Kentucky. Plant Dis. 74:761-766.

11. Huang, Y. H., and Hartman, G. L. 1998. Reaction of selected soybean genotypes to isolates of Fusarium solani f. sp. glycines and their culture filtrates. Plant Dis. 82:999-1002.

12. Iqbal, M. J., Yaegashi1, S., Ahsan, R., Shopinski, K.L., and, Lightfoot, D. A 2005. Root response to Fusarium solani f. sp. glycines: temporal accumulation of transcripts in partially resistant and susceptible soybean. Theor. Appl. Genet. 110:1429-1438.

13. Jin, H., Hartman, G. L., Nickell, C. D., and Widholm, J. M. 1996. Characterization and purification of a phytotoxin produced by Fusarium solani, the causal agent of soybean sudden death syndrome. Phytopathology 86:277282.

14. Jin, H., Hartman, G. L., Nickell, C. D., and Widholm, J. M. 1996. Phytotoxicity of culture filtrates from Fusarium solani, the causal agent of sudden death syndrome of soybean. Plant Dis. 80:922-927.

15. Kuruppu, P. U., Schneider, R. W., and Russin, J. S. 2004. Factors affecting soybean root colonization by Calonectria ilicicola and development of red crown rot following delayed planting. Plant Dis. 88:613-619.

16. Li, S., Hartman, G. L., and Chen, Y. 2009. Evaluation of aggressiveness of Fusarium virguliforme isolates that cause sudden death syndrome. J. Plant Pathol. 91:77-86.

17. Lozovaya, V. V., Lygin, A. V., Li, S., Hartman, G. L., and Widholm, J. M. 2004. Biochemical response of soybean roots to Fusarium solani f. sp. glycines infection. Crop Sci. 44:819-826.

18. Lozovaya, V. V., Lygin, A. V., Zernova, O. V., Li, S., Widholm, J. M., and Hartman, G. L. 2006. Lignin degradation by Fusarium solani f. sp. glycines. Plant Dis. 90:77-82

19. Navi, S. S., and Yang, X. B. 2008. Foliar symptom expression in association with early infection and xylem colonization by Fusarium virguliforme (formerly $F$. solani f. sp. glycines), the causal agent of soybean sudden death syndrome. Plant Health Progress doi: 10.1094/PHP-2008-0222-01-RS.

20. Phillips, D. V. 1972. Influence of photoperiod and of age and stage of development on brown stem rot of soybean. Phytopathology 62:1334-1337.

21. Ranathunge, K., Thomas, R. H., Fang, X., Peterson, C. A., Gijzen, M., and Bernards, M. A. 2008. Soybean root suberin and partial resistance to root rot caused by Phytophthora sojae. Phytopathology 98:1179-1189.

22. Rodriguez, A., Perestelo, F., Carnicero, A., Regalado, V., Perez, R., De la Fuente, G., and Falcon, M. A. 1996. Degradation of natural lignins and lignocellulosic substrates by soilinhabiting fungi imperfecti. FEMS Microbiol. Ecol. 21:213-219.

23. Roy, K. W., Lawrence, G. W., Hodges, H. H., McLean, K. S., and Killebrew, J. F. 1989. Sudden death syndrome of soybean: Fusarium solani as 
incitant and relation of Heterodera glycines to disease severity. Phytopathology 79:191-197.

24. Roy, K. W., Rupe, J. C., Hershman, D. E., and Abney, T. S. 1997. Sudden death syndrome of soybean. Plant Dis. 81:1100-1111.

25. Rupe, J. C. 1989. Frequency and pathogenicity of Fusarium solani recovered from soybean with sudden death syndrome. Plant Dis. 73:581-584.

26. Rupe, J. C., and Gbur, E. E., Jr. 1995. Effect of plant age, maturity group and the environment on the disease progress of sudden death syndrome of soybean. Plant Dis. 79:139-143.

27. Rupe, J. C., Gbur, E. E., and Marx, D. M. 1991. Cultivar response to sudden death syndrome of soybean. Plant Dis. 75:47-50.

28. Scherm, H., and Yang, X. B. 1996. Development of sudden death syndrome of soybean in relation to soil temperature and soil water matric potential. Phytopathology 86:642-649.

29. Smith, E. F., and Backman, P. A. 1989. Epidemiology of southern stem canker: effect of time of inoculum application on disease severity. Plant Dis. 73:464-468.

30. Thomas, R., Fang, X., Ranathunge, K., Anderson, T. R., Peterson, C. A., and Bernards, M. A. 2007. Soybean root suberin: anatomical distribution, chemical composition, and relationship to partial resistance to Phytophthora sojae. Plant Physiol. 144:299-311.

31. Verma, P. R., and Spurr, D. T. . 1987. Effect of plant age on temporal progression of common root-rot (Cochliobolus sativus) lesions on subcrown internodes of wheat and barley. Plant Soil 101:127-132.

32. Wrather, J. A., Kendig, S. R., Anand, S. C., Niblack, T. L., and Smith, G. S. 1995. Effects of tillage, cultivar, and planting date on percentage of soybean leaves with symptoms of sudden death syndrome. Plant Dis. 79:560-562.

33. Wrather, J. A., and Koenning S. R. 2009. Effects of diseases on soybean yields in the United States 1996 to 2007. Plant Health Progress doi: 10.1094/PHP-2009-0401-01-RS. 